\title{
Breast cancer following polyacrylamide hydrogel injection for breast augmentation: A case report
}

\author{
${\text { GANG } \mathrm{CHEN}^{1} \text {, YUJIA WANG }}^{2}$ and JIN-LONG HUANG ${ }^{1}$ \\ ${ }^{1}$ Department of Plastic Reconstructive Surgery and Medical Aesthetics, Nanjing University of TCM Affiliated Hospital, \\ Nanjing, Jiangsu 210029; ${ }^{2}$ Department of Oral and Maxillofacial Surgery, Nanjing Stomatological Hospital, \\ Nanjing University Medical School, Nanjing, Jiangsu 210008, P.R. China
}

Received July 3, 2015; Accepted October 27, 2015

DOI: $10.3892 / \mathrm{mco} .2016 .731$

\begin{abstract}
Polyacrylamide hydrogel (PAAG) has been used for several years as an injectable implant for augmentation mammoplasty in China. Although patients who received PAAG injections experienced a number of complications, breast cancer following PAAG injection has been reported only in two cases. In this report, we present a case of breast cancer following PAAG injection for breast augmentation. Our study demonstrated that PAAG injection may increase the risk of breast cancer development. Early-stage breast cancer detection is difficult, since the breast is covered with the indurated injected gel. Thus, PAAG injection for augmentation mammoplasty may negatively affect breast cancer diagnosis and prognosis.
\end{abstract}

\section{Introduction}

Polyacrylamide hydrogel (PAAG) is a jelly-like transparent substance containing $\sim 5 \%$ polyacrylamide and $95 \%$ water. PAAG was first manufactured in Ukraine in the late 1980s and introduced as a biomaterial for non-surgical breast augmentation. The polyacrylamide in this compound was found to be non-toxic and non-carcinogenic in several animal studies (1). PAAG injection for breast augmentation has been used for $\sim 10$ years in certain Chinese medical facilities. Although the clinical application of PAAG was banned on April 30, 2006 by the Chinese State Food and Drug Administration (2), the consequences and long-term complications of this gel may not appear until several decades later. The reported complications following PAAG injection for breast augmentation include induration, pain, swelling,

Correspondence to: Dr Jin-Long Huang, Department of Plastic Reconstructive Surgery and Medical Aesthetics, Nanjing University of TCM Affiliated Hospital, 155 Hanzhong Road, Nanjing, Jiangsu 210029, P.R. China

E-mail: xi_ao_2013@126.com

Key words: polyacrylamide hydrogel injection, breast cancer, augmentation mammoplasty, magnetic resonance imaging, ultrasonic, mammography infection, fever, aseptic inflammation, leakage, hematoma and gel migration (3). Due to these complications, a number of patients with mammoplasty augmentation history have requested removal of the injected gel or a simultaneous second augmentation as an alternative. However, in relation to the development of malignant breast tumors following PAAG injection, two cases of breast cancer occurring after injection of PAAG in augmented breasts were reported in 2009 (4).

In this report, we present a case of malignant breast tumor development following PAAG injection.

\section{Case report}

A 48-year-old woman underwent bilateral augmentation mammoplasty using injectable PAAG in 2003. Following mammary ptosis, the patient experienced hardening and a slow increase in the size of the left breast. In February, 2013, the left breast developed an infection, which was treated with antibiotic therapy in another facility.

When the patient was admitted to our department for further treatment in December, 2013, her vital signs were stable but her left breast appeared to be sagging, deformed and enlarged compared with her right breast. The patient's areolar diameter bilaterally was $\sim 4.5 \mathrm{~cm}$ and the nipple diameter was $\sim 1 \mathrm{~cm}$. No nipple discharge was observed (Fig. 1). On palpation, the left breast felt relatively harder, with occasional pricking on compression. There was no palpable mass in either breast. The blood biochemistry, six blood coagulation tests, electrocardiography and chest radiography examinations were normal. Magnetic resonance imaging (MRI) revealed post-injection augmented breasts and abnormal signals of an indeterminate nature on the left breast (Fig. 2). Although mammary gland hyperplasia was considered possible, other diseases should be excluded prior to diagnosis. The lymph nodes in the left axilla were enlarged. Aspiration of the injected PAAG from both breasts and biopsy of the inflamed mass in the left breast were performed. Over $300 \mathrm{ml}$ of injected PAAG were aspirated from each breast. The histological examination revealed fibrous breast tissue, with a silicon-like material.

Following surgery, an indurated mass appeared in the patient's left breast, which continued to grow to the size of a palm, without causing any discomfort. The patient was hospitalized again in March, 2014 and her vital signs were stable. 


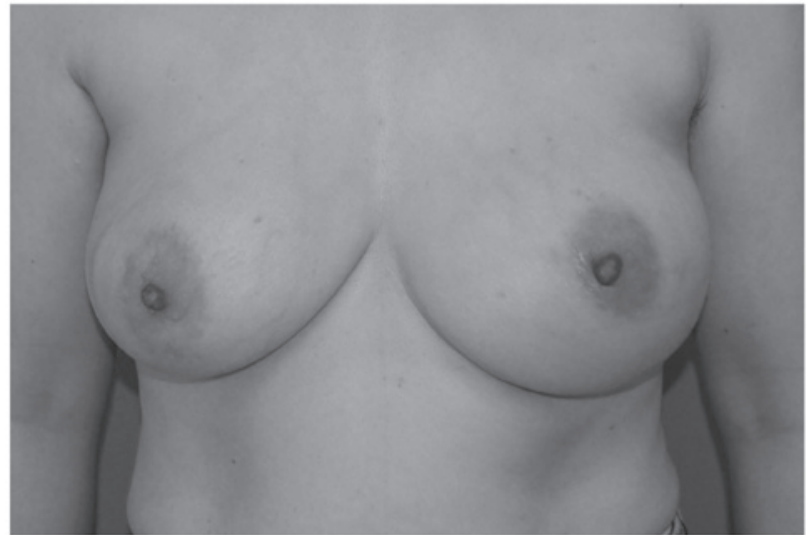

Figure 1. Prior to the first surgical treatment, the patient's left breast was saggy, deformed and enlarged compared with her right breast. The areolar diameter was $\sim 4.5 \mathrm{~cm}$ bilaterally, and the nipple diameter was $\sim 1 \mathrm{~cm}$. No nipple discharge was observed.

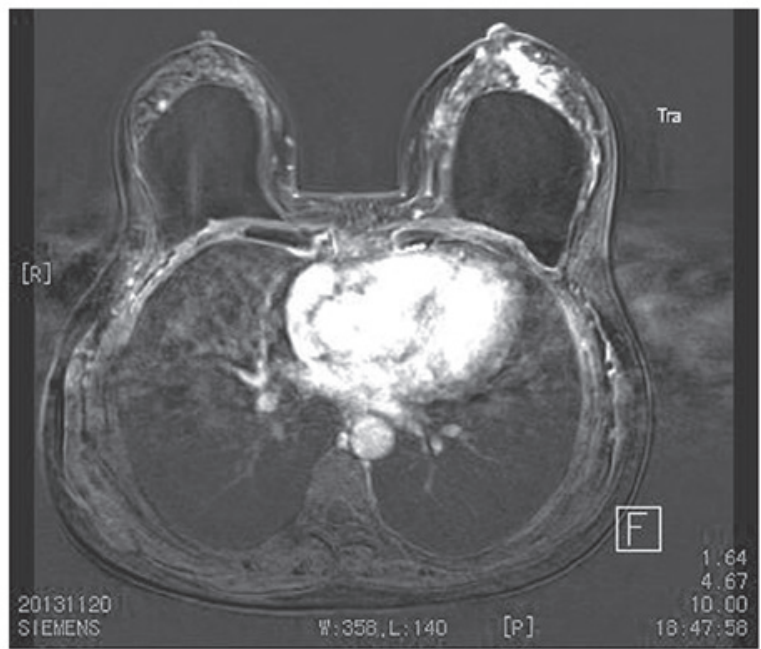

Figure 2. Magnetic resonance imaging during the first hospitalization: Post-injection augmentation mammoplasty changes, with abnormal signal intensity in the left breast and enlarged lymph nodes in the left axilla.

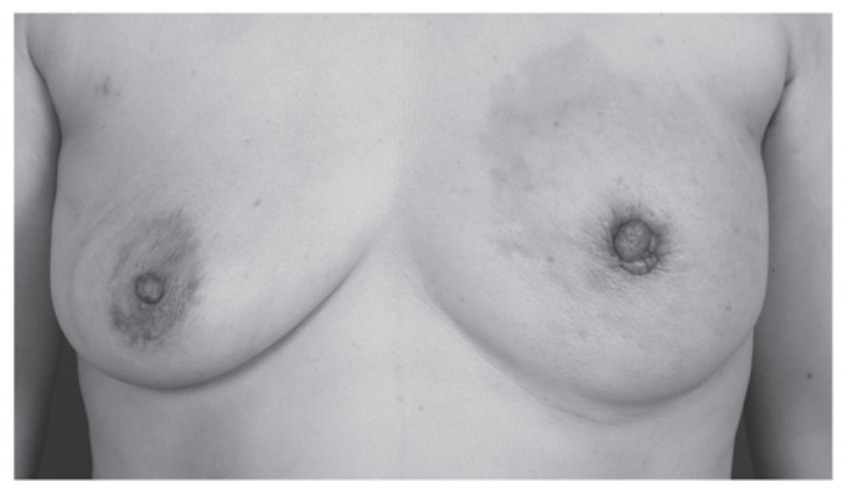

Figure 3. Prior to the second surgical treatment: A 5-cm skin patch surrounding the left nipple appeared erythematous, mildly puffy and pitted, resembling orange peel, without ulceration or high skin temperature.

A 5-cm patch of skin surrounding the left nipple appeared to be erythematous, with fading of the color on compression. The skin in this area was mildly puffy and pitted, resembling

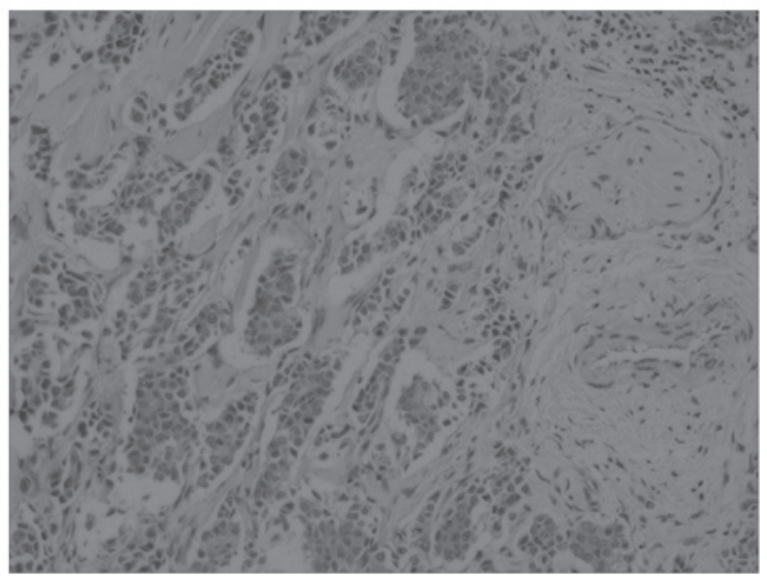

Figure 4. Histological examination: Stage II-III invasive breast cancer.

an orange peel. There were no ulcers and the patient's skin temperature was normal (Fig. 3). On examination, a hard, painless, ill-defined, fixed, 6x10-cm flaky mass was observed in the patient's left breast, accompanied by a mild depression of the left nipple. No regional enlarged lymph nodes were detected in either axilla. The tumor was excised for histopathological examination and the results revealed infiltration by stage II-III invasive breast carcinoma (Fig. 4). The immunohistological findings of the tumor were as follows: Estrogen receptor (-), progesterone receptor (-), Cerb-B2 (++), local Ki-67 60\% (+), high-molecular weight CK (-), CK5/6 (-), scattered calponin (+), P63 (-) and smooth muscle actin (+). The patient was advised to undergo breast surgery and was referred to the oncology department of the hospital for further treatment. No reccurence or distant metastasis is reported to date.

\section{Discussion}

In this study, we present a case that demonstrates the correlation between PAAG injection and breast cancer development. Since breast augmentation by injection is considered to be cosmetic surgery, pre-set control and randomized research methods were not feasible in the present study.

A previous study has indicated that PAAG increases the mRNA expression of the c-Myc proto-oncogene, which may inhibit the growth and cause apoptosis of human fibroblasts and alter the physical parameters of cells, such as their size and granularity (5). This might increase the risk of breast cancer, but further evidence is needed.

The patient was first hospitalized with a major complaint of post-PAAG injection complications rather than the occurrence of a tumor. During the first hospitalization, no typical complaints, symptoms or signs of cancer (e.g., orange peel-like skin; hard, ill-defined and fixed lump; or enlarged lymph nodes in the axilla) were reported. Although certain signs and symptoms of a breast tumor were observed during the patient's second hospitalization, overlooking the possibility of cancer is possible due to the limited number of case reports and studies on the correlation between PAAG injection and breast cancer.

The currently available evidence indicate that breast implants do not induce local or systemic disease, particularly 
breast cancer (6-9). However, implants may impair early-stage breast cancer identification by mammography, as cosmetic breast implants are radio-opaque and impair breast tissue visualization $(8,10,11)$. Women with cosmetic breast implants may present with late-stage tumors upon breast cancer diagnosis (12).

Breast lump formation resulting from gel collection in mammary tissues is the most common complication in PAAG-injected augmentation mammoplasty patients. Compared with prosthetic augmentation mammoplasty (PAM) patients with silicone implants, early diagnosis of breast tumors is more difficult in PAAG-injected patients, as differentiating between the injected hydrogel lump and a possible neoplasm on palpation is very difficult.

Recent imaging techniques allow detection of breast cancer in breasts with implants, even in cases without a palpable mass (13). However, mammography cannot accurately assess the postoperative status of PAAG-injected breasts (14).

Ultrasonography and MRI are more efficient for malignancy detection in PAM patients with implants compared with mammography (15-19). Ultrasonography is the first step in investigating symptomatic patients with augmented breasts aged $<40$ years, in order to evaluate the breast or rule out pathologies associated with the implant. This imaging technique is also used as a complement to mammography in patients aged $>40$ years who present with pathological findings on screening or diagnostic mammography $(16,17)$.

MRI allows examination of breast tissue surrounding the implant and exhibits a higher sensitivity compared with mammography $(18,19)$. Ultrasonography must be considered as a routine adjunctive screening method for PAAG-injected patients. When ultrasound detects abnormal pathologies associated with the implant, MRI may be used as a highly reliable method for accurately detecting masses in augmented breasts (20-22).

The results of our study are of great clinical significance and suggest that physicians must be more aware of possible breast cancer. A patient's medical history, physical examination results and multiple imaging findings must be comprehensively analyzed to avoid a misdiagnosis. More effective methods must be established to visualize the lesions and distinguish injected materials and inflamed masses from tumors. During surgery, multiple tissue samples must be drawn from suspicious nodules and sent for frozen section evaluation. No significant difference in surgical intervention and prognosis is reported between PAM and non-PAM patients with breast cancer (23). However, based on the patient's presentation, treatment and prognosis, more advanced strategies must be designed for breast cancer patients with PAAG injection mammoplasty.

PAAG injection augmentation may be correlated with breast cancer, and breast cancer diagnosis in post-injection augmentation mammoplasty patients may be difficult. While ultrasonic and MRI methods have been used to detect breast cancer, the sensitivity and accuracy of these techniques for distinguishing between injected gel lumps and tumors must be improved to identify tumors at the early stages of development. Great caution and multiple methods may also be required to avoid misdiagnosis.

\section{References}

1. Amended final report on the safety assessment of polyacrylamide and acrylamide residues in cosmetics. Int J Toxicol 24 (Suppl 2): S21-S50, 2005

2. State Food and Drug Administration: About prohibited the use of polyacrylamide hydrogel (injection) warning. http://www.sda. gov.cn/WS01/CL0493/93434.html. Accessed May 10, 2015.

3. Luo SK, Chen GP, Sun ZS and Cheng NX: Our strategy in complication management of augmentation mammaplasty with polyacrylamide hydrogel injection in 235 patients. J Plast Reconstr Aes 64: 731-737, 2011.

4. Cheng NX, Liu LG, Hui L, Chen YL and Xu SL: Breast cancer following augmentation mammaplasty with polyacrylamide hydrogel (PAAG) injection. Aesthetic Plast Surg 33: 563-569, 2009.

5. Xi TF, Fan CX, Feng XM, Wan ZY, Wang CR and Chou LL: Cytotoxicity and altered c-myc gene expression by medical polyacrylamide hydrogel. J Biomed Mater Res A 78: 283-290, 2006.

6. Deapen DM, Hirsch EM and Brody GS: Cancer risk among Los Angeles women with cosmetic breast implants. Plast Reconstr Surg 119: 1987-1992, 2007.

7. Deapen DM and Brody GS: Augmentation mammaplasty and breast cancer: A 5-year update of the Los Angeles study. Plast Reconstr Surg 89: 660-665, 1992.

8. Hoshaw SJ, Klein PJ, Clark BD, Cook RR and Perkins LL: Breast implants and cancer: Causation, delayed detection and survival. Plast Reconstr Surg 107: 1393-1408, 2001.

9. Brinton LA, Lubin JH, Burich MC, Colton T, Brown SL and Hoover RN: Breast cancer following augmentation mammoplasty (United States). Cancer Causes Control 11: 819-827, 2000.

10. Handel N, Silverstein MJ, Gamagami P, Jensen JA and Collins A: Factors affecting mammographic visualization of the breast after augmentation mammaplasty. Jama 268: 1913-1917, 1992.

11. Fajardo LL, Harvey JA, Mcaleese KA, Roberts CC and Granstrom P: Breast-cancer diagnosis in women with subglandular silicone gel-filled augmentation implants. Radiology 194: 859-862, 1995.

12. Lavigne E, Holowaty EJ, Pan SY, Villeneuve PJ, Johnson KC, Fergusson DA, Morrison $\mathrm{H}$ and Brisson J: Breast cancer detection and survival among women with cosmetic breast implants: Systematic review and meta-analysis of observational studies. BMJ 346: f2399, 2013.

13. Clark CPIII,Peters GN and O'Brien KM: Cancer in the augmented breast. Diagnosis and prognosis. Cancer 72: 2170-2174, 1993.

14. Peng DHLM, Liu LG and Gong JS: Mammographic appearance of postoperative augmentation mammoplasty with hydrogel. Chin J Radiol 40: 354-356, 2006.

15. Leibman AJ and Kruse B: Breast cancer: Mammographic and sonographic findings after augmentation mammoplasty. Radiology 174: 195-198, 1990.

16. Dhamanaskar KP, Muradali D, Kulkarni SR, Bukhanov K, Pantazi SC and Wilson C: MRI directed ultrasound: A cost-effective method for diagnosis and intervention in breast imaging. Radiology 225: 653-653, 2002.

17. Esen G and Olgun DC: Ultrasonography of the postsurgical breast including implants. Ultrasound Clinics 3: 295-329, 2008.

18. Juanpere S, Perez E, Huc O, Motos N, Pont J and Pedraza S: Imaging of breast implants - a pictorial review. Insights Imaging 2: 653-670, 2011.

19. Orel SG and Schnall MD: MR imaging of the breast for the detection, diagnosis and staging of breast cancer. Radiology 220 : 13-30, 2001.

20. Chung KC, Greenfield ML and Walters M: Decision-analysis methodology in the work-up of women with suspected silicone breast implant rupture. Plast Reconstr Surg 102: 689-695, 1998.

21. Hold PM, Alam S, Pilbrow WJ, Kelly JF, Everitt EM, Dhital SK and Juma A: How should we investigate breast implant rupture? Breast J 18: 253-256, 2012.

22. Peng HL, Wu CC, Choi WM, Hui HS, Lu TN and Chen LK: Breast cancer detection using magnetic resonance imaging in breasts injected with liquid silicone. Plast Reconstr Surg 104: 2116-2120, 1999 .

23. Handel N: The effect of silicone implants on the diagnosis, prognosis and treatment of breast cancer. Plast Reconstr Surg 120 (7 Suppl 1): S81-S93, 2007. 